\title{
Charge-sign dependent modulation in the heliosphere over a 22-year cycle
}

\author{
S. E. S. Ferreira ${ }^{1,3}$, M. S. Potgieter ${ }^{1}$, B. Heber ${ }^{2}$, and H. Fichtner ${ }^{3}$ \\ ${ }^{1}$ Unit for Space Physics, School of Physics, Potchefstroom University for CHE, 2520 Potchefstroom, South Africa \\ ${ }^{2}$ Fachbereich Physik, Universitt Osnabrück, 49069 Osnabrück, Germany \\ ${ }^{3}$ Institut für Theoretische Physik, Lehrstuhl IV: Weltraum und Astrophysik, Ruhr-Universität Bochum, Germany
}

Received: 7 November 2002 - Revised: 10 March 2003 - Accepted: 19 March 2003

\begin{abstract}
A time-dependent model based on a numerical solution of Parker's transport equation is used to model the modulation of cosmic ray protons, electrons and helium for full 11-year and 22-year modulation cycles using a compound approach. This approach incorporates the concept of propagating diffusion barriers based on global increases in the heliospheric magnetic field as they propagate from the Sun throughout the heliosphere, combined with gradient, curvature and current sheet drifts and the other basic modulation mechanisms. The model results are compared to the observed 11-year and 22-year cycles for 1.2 GV electrons and 1.2 GV Helium at Earth for the period 1975-1998. The model solutions are also compared to the observed chargesign dependent modulation along Ulysses' trajectory for the period 1990-1998. This compound approach to long-term modulation, especially charge-sign dependent modulation, is found to be remarkably successful. It is shown that the model can easily account for the latitude dependence for cosmic ray protons and the lack thereof for cosmic ray electrons by assuming large perpendicular diffusion in the polar direction. This approach contributes to an improved understanding of how diffusion and drifts vary from solar minimum to maximum modulation, and what the time-dependence of the heliospheric diffusion coefficients may be.
\end{abstract}

Key words. Interplanetary physics (energetic particles; cosmic rays; general or miscellaneous)

\section{Introduction}

Describing the heliospheric modulation of cosmic rays successfully over long time periods, including periods of large to maximum solar modulation, where step decreases in intensities are observed, requires some form of propagating diffusion barriers, as was first introduced by Perko and Fisk (1983) - see also le Roux and Potgieter (1989). The ex-

Correspondence to: S. E. S. Ferreira

(fsksesf@puknet.puk.ac.za) treme form of these diffusion barriers is called global merged interaction regions (GMIRs), as introduced by Burlaga et al. (1993). Equally important to long-term cosmic ray modulation are gradient, curvature and current sheet drifts (Jokipii et al., 1977), as confirmed by comprehensive modeling done by Potgieter et al. (1993) and le Roux and Potgieter (1995). For reviews on long-term modulation modeling, see Potgieter (1993, 1995, 1997), Potgieter et al. (2001) and Ferreira and Potgieter (2003). These authors found that it was possible to simulate, to the first order, a complete 11-year proton modulation cycle by including a combination of drifts and GMIRs in a comprehensive time-dependent model. These simulations were generally done for radial distances larger than $20 \mathrm{AU}$, allowing enough time for merging of corotating structures to take place. More recently, Cane et al. (1999) and Wibberenz and Cane (2000) argued that the cosmic ray step decreases observed at Earth cannot be caused by GMIRs because these decreases occurred before any GMIRs could form beyond 10-20 AU. Instead, they suggested that time-dependent global changes in the heliospheric magnetic field (HMF) might be responsible for long-term modulation. This approach was modeled and tested by Ferreira (2002) by changing all the diffusion coefficients in a full time-dependent model to reflect the time-dependent changes in the measured HMF magnitude at Earth. These changes were propagated outwards at the solar wind speed to form effective propagating diffusive barriers throughout the heliosphere, changing with the solar cycle. This approach could simulate an 11-year modulation cycle successfully for neutron monitor cosmic ray observations at, for example, $16 \mathrm{GV}$. For rigidities $<5 \mathrm{GV}$ it resulted in far less modulation than what was observed, so that a modified approach was proposed, called the compound approach. This approach combines the effects of the global changes in the HMF magnitude with drifts, therefore also time dependent current sheet "tilt angles", to establish realistic time-dependent diffusion coefficients. The compound model was described by Ferreira (2002) - see also Potgieter and Ferreira (2001) and Potgieter et al. (2001). In this work it is used to model the long- 
term modulation of $1.2 \mathrm{GV}$ electron and Helium intensities at Earth and the $2.5 \mathrm{GV}$ electron and proton intensities along Ulysses' trajectory, in order to study charge-sign dependent modulation over a full 22-year cycle - see also Burger and Potgieter (1999) and Potgieter et al. (2001). For this purpose the time-dependence of the heliospheric diffusion coefficients is computed, illustrating how diffusion and drifts change from solar minimum to maximum modulation.

\section{Model and parameters}

The model is based on a numerical solution of Parker's (1965) time-dependent transport equation:

$$
\begin{aligned}
\frac{\partial f}{\partial t} & =-\left(\mathbf{V}+\left\langle\mathbf{v}_{D}\right\rangle\right) \cdot \nabla f+\nabla \cdot\left(\mathbf{K}_{s} \cdot \nabla f\right) \\
& +\frac{1}{3}(\nabla \cdot \mathbf{V}) \frac{\partial f}{\partial \ln P}+J_{\text {source }}
\end{aligned}
$$

where $f(\mathbf{r}, P, t)$ is the cosmic ray distribution function; $P$ is rigidity, $\mathbf{r}$ is position, $\mathbf{V}$ is the solar wind velocity and $t$ is time. Terms on the right-hand side represent convection, gradient, and curvature drifts, diffusion, adiabatic energy changes, and a source function, respectively. The symmetric tensor $\mathbf{K}_{s}$ consists of a parallel diffusion coefficient $\left(K_{\|}\right)$and two perpendicular diffusion coefficients, in the radial direction $\left(K_{\perp r}\right)$ and in the polar direction $\left(K_{\perp \theta}\right)$. In Eq. (1) the pitch angle averaged guiding center drift velocity for a near isotropic cosmic ray distribution is given by $\left\langle\mathbf{v}_{D}\right\rangle=\nabla \times\left(K_{A} \mathbf{e}_{B}\right)$, with $\mathbf{e}_{B}=\mathbf{B} / B_{m}$ and $B_{m}$ the magnitude of the modified background HMF.

Equation (1) was solved time-dependently based on the numerical procedure of le Roux and Potgieter (1995) for both the so-called $A>0(\sim 1970-1980 ; \sim 1990-2001)$ and $A<0$ epochs $(\sim 1980-1990 ; \sim 2001)$, using as time-dependent input parameters the time varying "Hoeksema tilt angles", $\alpha$ (see Wilcox Solar Observatory: http://quake.stanford.edu/) illustrated in Fig. 1 (bottom panel), and measured HMF values at Earth shown in the middle panel of Fig. 1 (see NSSDC COHOWeb: http://nssdc.gfc.nasa.gov/cohoweb). Two different Hoeksema-models exist for calculating $\alpha$; the "classic" model uses a line-of-sight boundary condition and the "new" model uses a radial boundary condition at the photosphere (Hoeksema, 1992). Ferreira (2002) found that the $\alpha$ with the smallest rate of change over a period of decreasing or increasing solar activity provides the best compatibility with cosmic ray observations - see also Ferreira and Potgieter (2003). Therefore in this work, where the modulation of cosmic rays is explored over 22 years, $\alpha$ 's corresponding to the "new" model are used for periods of increasing solar activity (1976.0-1979.9, 1987.4-1990.0 and 1995.52000.0) and $\alpha$ 's corresponding to the "classic" model are used for periods of decreasing solar activity (1979.9-1987.4 and 1990.0-1995.5).

The outer modulation boundary was assumed at $120 \mathrm{AU}$, where the different local interstellar spectra were specified. The solar wind speed $\mathrm{V}$ was assumed to change from
$400 \mathrm{~km} \mathrm{~s}^{-1}$ in the equatorial plane (polar angle $\theta=90^{\circ}$ ) to a maximum of $800 \mathrm{~km} \mathrm{~s}^{-1}$ when $\theta \leq 60^{\circ}$ and $\theta \geq 120^{\circ}$ for solar minimum conditions (McComas et al., 2001), while for solar maximum $V=400 \mathrm{~km} \mathrm{~s}^{-1}$ for all polar angles. The effects of a time-dependence in the latitude dependence of the solar wind speed on the modulation of cosmic rays, in particular low energy electrons, are illustrated in Ferreira et al. (2003).

For the diffusion coefficients we assume:

$K_{\|}=f_{1}(r, P, t) f_{2}(t) \sigma$ with $f_{2}(t)=\left(\frac{B_{0}}{B(t)}\right)^{n}$,

where $f_{1}(r, P, t)$ is a function determining the rigidity and spatial dependence as given by Ferreira et al. (2001), with $\sigma=1.3$ for $A<0$ epochs, when positive particles drift mainly in along the current sheet, and $\sigma=1.0$ for $A>0$ epochs when positive particles drift in over the poles. This gives different diffusion coefficients for alternate solar cycles in qualitative agreement with Reinecke et al. (1996) and Potgieter (2000), who found that these coefficients were different for consecutive solar minimum periods. This might also indicate that an additional charge-sign dependent mechanism could be contributing to modulation such as magnetic helicity (e.g. Burger et al., 1997). The function $f_{2}(t)$, with $B_{0}=5 \mathrm{nT}$, determines the time-dependence of all the diffusion coefficients as a function of the measured HMF at Earth, $B(t)$, which is interpreted to be an elementary representation of turbulence over a solar cycle. Potgieter and Ferreira (2001) illustrated that $n=1$ in Eq. (2) resulted in realistic computed modulation at neutron monitor rigidities, which means that changing the diffusion coefficients with time by a factor of $\sim 2$, corresponding to changes in the HMF magnitude from solar minimum to maximum, is sufficient at these high rigidities. For lower rigidities, when the computed intensities were compared to observed $1.2 \mathrm{GV}$ electron and Helium intensities at Earth, this approach did not reproduce the observed modulation amplitudes over 11 years; even values of $n \approx 3.0$ could not reproduce the required amplitude. These authors concluded that $n$ could not be a constant and had to change with time (and rigidity), where this time dependence must be related to solar activity. From a drift point of view, the obvious choice was the time-varying $\alpha$. The compound approach for long-term cosmic ray modulation was consequently proposed by assuming $n=\alpha / \alpha_{0}$, with $\alpha_{0}=11$, which may vary with rigidity. Using this form means that $n$ is small $(n \rightarrow 0)$ for minimum modulation, but increases with increasing solar activity $(n=2 \rightarrow 5)$. The larger $n$ is made, the larger the temporal changes in the diffusion coefficients become, simulating essentially a series of propagating diffusive barriers of changing magnitude. In this work the same compound model is used, and in the top panel of Fig. 1, the time dependence in the diffusion coefficients $f_{2}(t)$ is shown for the period 1975 up to 2000. Evidently, the diffusion coefficients are larger ( $\sim$ a factor of 10 or more depending on the rigidity) at solar minimum than at solar maximum - see also Cummings and Stone (2001) - and are highly time-dependent. These time 


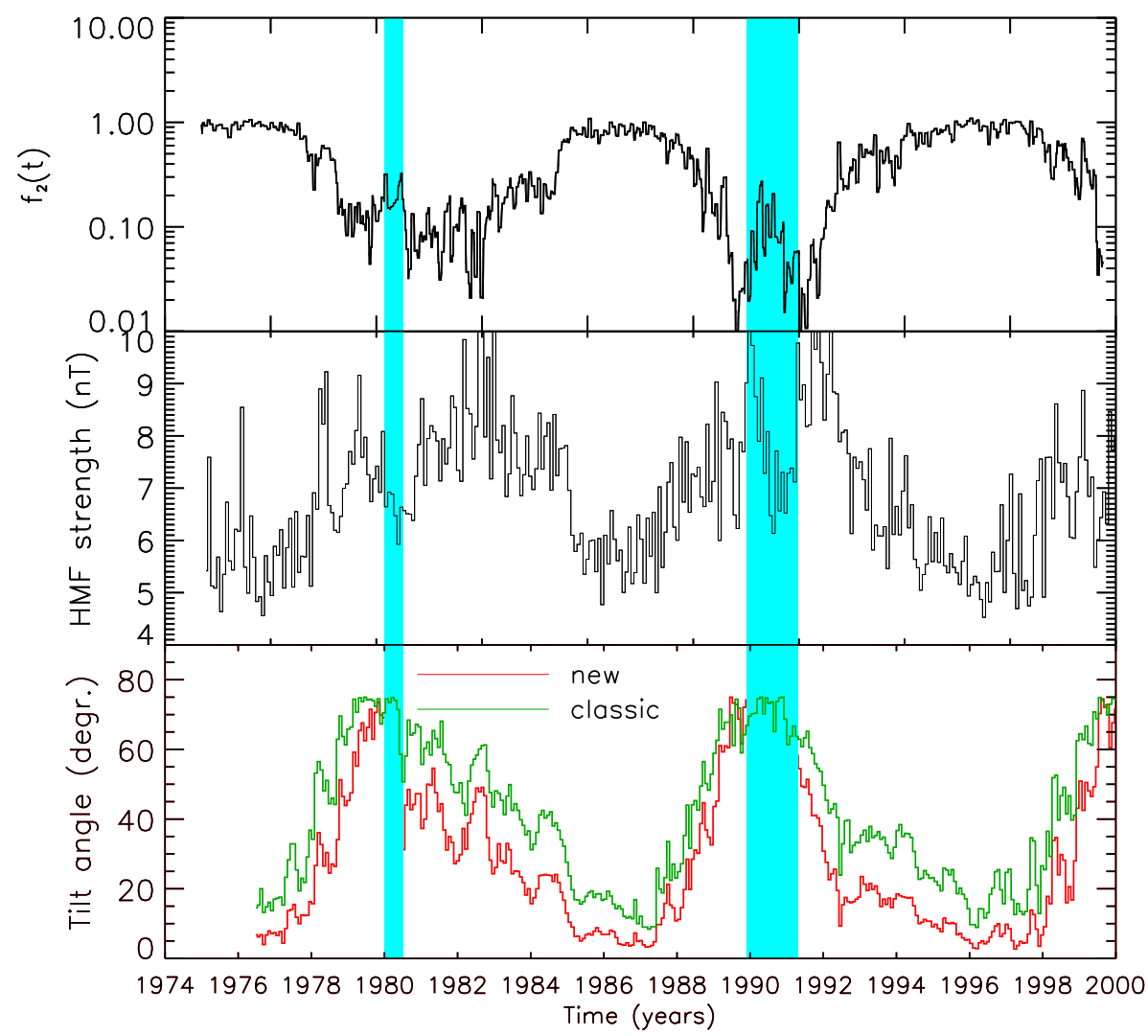

Fig. 1. Top panel: The function $f_{2}(t)$ in Eq. (2) represents the time-dependence of all the heliospheric diffusion coefficients for the period 1975 up to 2000. This function combines the effects of the observed global changes in the heliospheric magnetic field (HMF) magnitude (middle panel; NSSDC COHOWeb: http://nssdc.gfc.nasa.gov/cohoweb) with drifts, therefore also time dependent current sheet "tilt angles" (bottom panel; Wilcox Solar Observatory: http://quake.stanford.edu/), to establish the time-dependence of the diffusion coefficients.

dependent changes are propagated outwards into the heliosphere at the solar wind speed, causing time-dependent diffusive barriers to move from $1 \mathrm{AU}$ and beyond. Because these diffusive barriers play only an important role for intermediate to larger solar activity and to avoid numerical problems, their propagation speed into the heliosphere is chosen as the slow solar wind speed. However, these barriers may also merge, eventually, but no merging was allowed for this work. Also, the spatially two-dimensional nature of the model means that we average the cosmic ray intensities over one solar rotation. Therefore, the effects of recurrent features of the solar wind speed, like corotating interaction regions on the modulation of high energy cosmic rays are not considered (see Kissmann et al., 2003). However, the effects of merged corotating interaction regions on the modulation of cosmic rays was studied by Potgieter et al. (1993) and Potgieter and le Roux (1994). They confirmed that these merged interaction regions contribute little to long-term modulation and the concept of global merged interaction regions (propagating diffusive barriers) is needed to simulate cosmic ray modulation successfully.

Concerning perpendicular diffusion, no exact theory exists to adequately describe it up to date (for a comprehensive discussion, see le Roux et al., 1999). Therefore, it has become standard practice when using numerical modulation models to scale $K_{\perp}$ as $K_{\|}$(e.g. Kóta and Jokipii, 1998; Burger et al., 2000). For a theoretical motivation see le Roux et al. (1999). For $K_{\perp r}$ and $K_{\perp \theta}$ we assumed that

$K_{\perp r}=0.02\left(\frac{P}{P_{0}}\right)^{0.3} K_{\|}$

and $K_{\perp \theta} / K_{\|}=b F(\theta)$

with $F(\theta)=A^{+}+A^{-} \tanh \left[\frac{1}{\Delta \theta}\left(\theta_{A}-90^{\circ}+\theta_{F}\right)\right]$.

Here, $P_{o}=1 \mathrm{GV}$ and $b=0.03$. This results in $K_{\perp r} / K_{\|} \geq$ 0.02 for $P \geq 1 \mathrm{GV}$ and $K_{\perp \theta} / K_{\|}=0.03$ (in the equatorial plane) as required by the simulations done by Giacalone and Jokipii (1999). Furthermore, Burger et al. (2000) illustrated that, in order to produce the correct magnitude and rigidity dependence of the observed latitudinal cosmic ray proton density gradient by Ulysses, enhanced latitudinal transport is required (see also Potgieter et al., 1997). This is accomplished by increasing $K_{\perp \theta}$ toward the poles by a factor $d=8$ with respect to the value in the equatorial plane by assuming the function $F(\theta)$ in Eq. (3), where $A^{ \pm}=(d \pm 1) / 2, \Delta \theta=1 / 8, \theta_{A}=\theta$ and $\theta_{F}=35^{\circ}$ for 

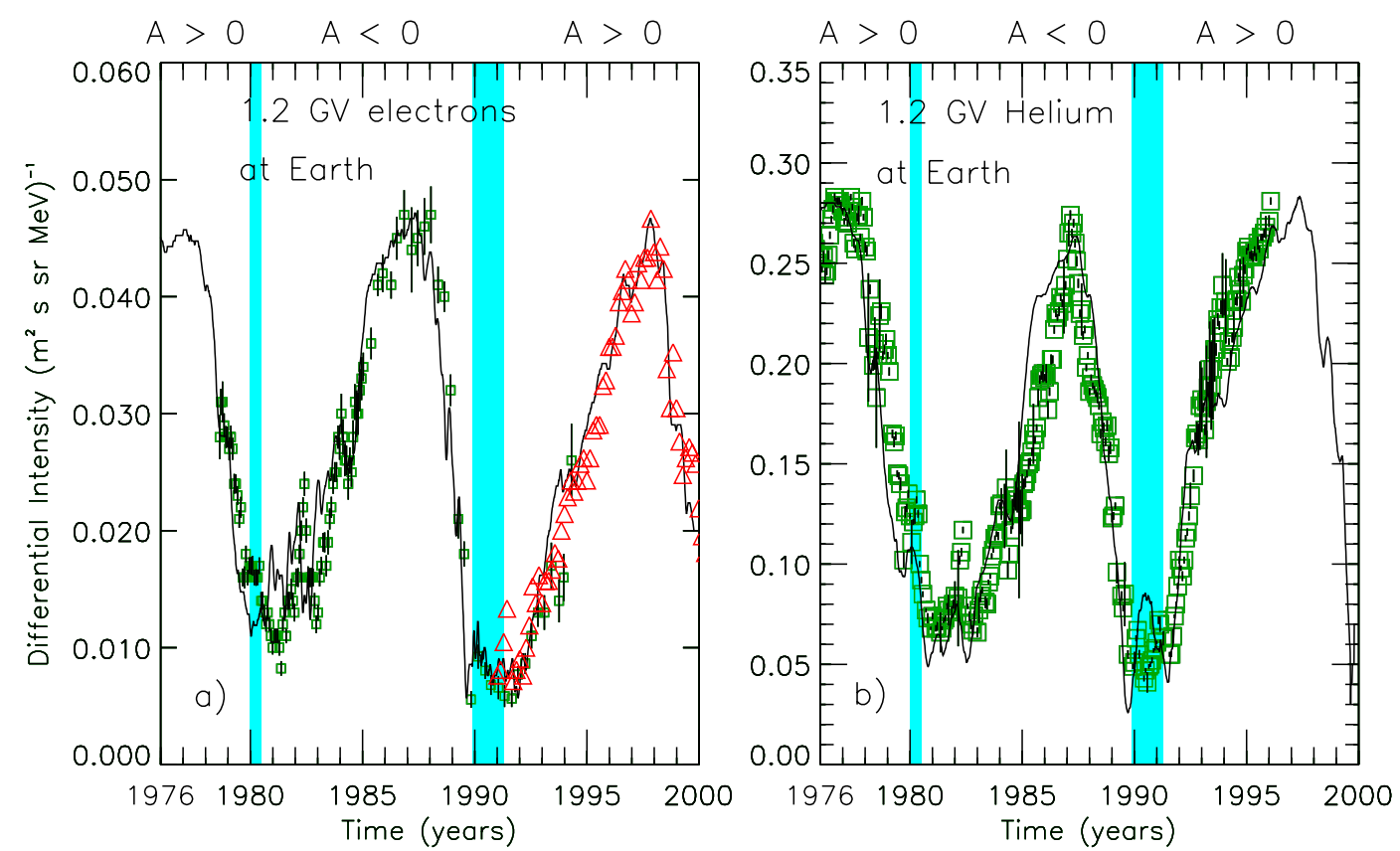

Fig. 2. (a) Computed $1.2 \mathrm{GeV}$ electron intensity at Earth compared to observations from ISEE3/ICE (e.g. Clem et al., 1996; Evenson, 1998) and Ulysses (e.g. Heber et al., 2002; Clem et al., 2002). (b) Computed 1.2 GV Helium intensity at Earth compared to observations from IMP (McDonald, 1998; McDonald et al., 2001).

$\theta \leq 90^{\circ}$ while for $\theta>90^{\circ}, \theta_{A}=180^{\circ}-\theta$. A justification of this increase in $K_{\perp \theta}$ toward the polar regions was given, for example by Burger et al. (2000). These arguments are based on Ulysses' measurements which show the variance in the transverse and normal directions of the HMF to increase more than in the radial direction, resulting in larger diffusion in this direction. Furthermore, in a Fisk-type HMF (Fisk, 1996), which is probably a more realistic HMF geometry for solar minimum conditions, latitudinal transport is supposedly more effective than in a Parker field, and to account for this effect, $K_{\perp \theta}$ is enhanced toward the polar regions when a Parker HMF is used. The function $F(\theta)$ is shown in Fig. 4c with $d=8$ and $\theta_{F}=35^{\circ}$, and with $d=6$ and $\theta_{F}=15^{\circ}$. The effects of these two scenarios on model computations will be shown and discussed in Fig. 4. Because both the perpendicular diffusion coefficients scale as $K_{\|}$, their values are also changed time-dependently, as shown in Fig. 1.

The drift coefficient is changed time-dependently as follows:

$K_{A}=\left(K_{A}\right)_{0} \frac{K_{\mathrm{drift}}(P)}{3 B_{m}} f_{2}(t)$

with $K_{\mathrm{drift}}(P)=\beta P \frac{D_{\mathrm{fak}} P^{2}}{D_{\mathrm{fak}} P^{2}+1}$

and with $B_{m}$ the Parker HMF modified only in the heliospheric polar regions similar to what Jokipii and Kóta (1989) used; $D_{\text {fak }}=10.0$ in units of (rigidity) ${ }^{-2}$, which causes drifts to be somewhat reduced at lower rigidities, as explained by Burger et al. (2000); $\left(K_{A}\right)_{0}=1.0$, and $\beta$ is the ratio between the particle speed and the speed of light. This means that $f_{2}(t)$, as shown in Fig. 1 , together with $\left(K_{A}\right)_{0}$, is also indicative of the drifts required over a full 22-year cycle. During solar minimum periods, drifts are obviously large, varying between $80 \%$ and $100 \%$ for at least three years around every minimum. For $A<0$ cycles, around, for example,1985, drifts drop sharply with increased modulation but not for the $A>0$ cycle, around, for example, 1997, which is the cause of the well-known flat-like versus sharp intensitytime profiles in cosmic ray observations of all energies, respectively, during $A>0$ and $A<0$ polarity cycles. During solar maximum conditions in 1979-1982, drifts reduced to less than $10 \%$ for most of the period, while during the 19891992 solar maximum, it reduced to essentially $0 \%$ for two relatively short periods, and remained below $10 \%$ for three out of the four years. In the next section the model solutions will be shown to illustrate that this approach and drift levels are consistent with the data for the two maximum epochs.

\section{Results and discussions}

Figure 2 shows the results of the compound modelling approach where the computed intensities are compared, respectively, to the $1.2 \mathrm{GeV}$ electron observations and $1.2 \mathrm{GV}$ Helium observations at Earth (e.g. Clem et al., 1996; Evenson, 1998; McDonald, 1998; McDonald et al., 2001; Clem et al., 2002; Heber et al., 2002). As illustrated, this approach produces the correct modulation amplitude and most of the modulation steps in the observations. Some of these simulated steps do not have the correct magnitude and phase, indicating that some refinement of this approach is still needed, allowing, for example, for some global merging of the prop- 

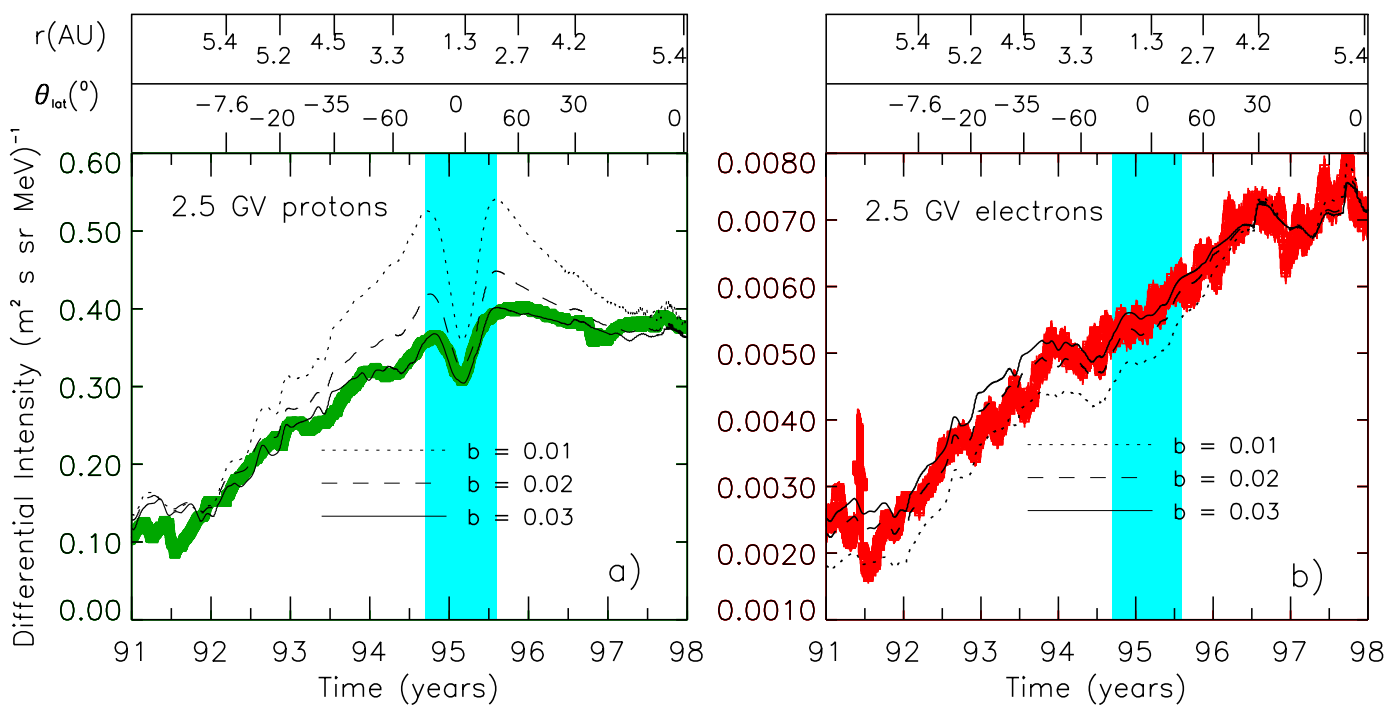

Fig. 3. (a) Computed $2.5 \mathrm{GV}$ proton intensity and (b) $2.5 \mathrm{GV}$ electron intensity along Ulysses' trajectory in comparison with $2.5 \mathrm{GV}$ data from the Ulysses/KET instrument (Heber et al., 1996, 1999, 2002).

agating diffusion barriers. However, the gratifying aspect of these results is that solar maximum modulation could indeed be largely reproduced for different cosmic ray species using a relatively simple concept, while maintaining the major modulation features during solar minimum, like the flatter modulation profile for electrons (Helium) in 1987 (1997), but a sharper profile for 1997 (1987). It was illustrated by Ferreira (2002) that this model could also produce realistic cosmic ray modulation in the outer heliosphere.

The observed latitudinal gradients for cosmic ray proton are a major challenge for modelling. These gradients are significantly smaller as observed by Ulysses for the $A>0$ polarity cycle (see the review by Heber and Potgieter, 2000) than predicted by classical drift models (e.g. Haasbroek and Potgieter, 1995). For $A>0$ polarity cycles positive particles, like cosmic ray protons, drift in from the poles of the heliosphere to the Sun and outward along the heliospheric current sheet. For these periods large latitudinal gradients were calculated by drift-dominated models. However, comparing the model results to the Ulysses observations, which observed much smaller gradients, it became quickly evident that this was due to the overestimation of drifts in the polar regions of the heliosphere in these models (Potgieter et al., 1997). Also, when the clear effects of corotating interaction regions were observed (McKibben et al., 1995; Paizis et al., 1999) in cosmic ray intensities at high heliolatitudes, without accompanying structures in the solar wind and the HMF, it became obvious that effective latitudinal transport occurs in the heliosphere. It could not result from drifts, because then the latitudinal gradients would have been large. The obvious answer was in large perpendicular diffusion in the polar region, $K_{\perp \theta}$ (e.g. Kóta and Jokipii, 1998). Drifts effects can effectively be reduced by increasing $K_{\perp \theta}$ (Potgieter, 2000; Ferreira et al., 2000). The exact enhancement of this coefficient in the polar direction is still somewhat controversial and may change if a non-Parkerian HMF is used.

Encouraged by the results shown in Fig. 2, the compound concept is applied in Fig. 3 to illustrate the success of reducing drifts effectively through a large $K_{\perp \theta}$. In Fig. 3a the computed proton intensity is shown and in Fig. $3 b$ one sees the $2.5 \mathrm{GV}$ electron intensity along the Ulysses trajectory. Shown in comparison are the Ulysses/KET data (Heber et al., 2002). For both species three computed scenarios are shown corresponding to three assumptions of $b$ in Eq. (3), which gives the magnitude of $K_{\perp \theta}$ in the equatorial plane. In accordance with the observations, there are noticeable computed latitudinal gradients for the proton intensity, as is especially evident during the fast latitude scan in 1995, but almost none for the electron intensities. This feature has recently been studied in great detail with the availability of the Ulysses/KET simultaneous observations of electrons and protons (e.g. Heber et al., 1999). Comparing the model with the observations shows that $b=0.03$ in Eq. (4) does produce realistic latitudinal gradients for both species and that large $K_{\perp \theta}$ is indeed necessary to compute realistic charge-sign dependent modulation over the full period shown. Decreasing $K_{\perp \theta}$ by assuming $b=0.01$ in Eq. (4) obviously results in computed latitudinal gradients that are top large, because the drifts then become too large. It is also evident in Fig. 3 that decreasing $b$ results in a larger computed modulation amplitude, especially for the $2.5 \mathrm{GV}$ electrons, indicating that the compound approach is sensitive to this diffusion coefficient. The compound model is evidently simulating the 11 year modulation cycle in the inner heliosphere without global merged interaction regions.

In Fig. 4a the electron-to-proton ratio is calculated along Ulysses' trajectory and at Earth. Comparing the model computations of this ratio to the observations shows that the charge-sign dependence, as observed by Ulysses, could also easily be explained by the compound model. The presence 


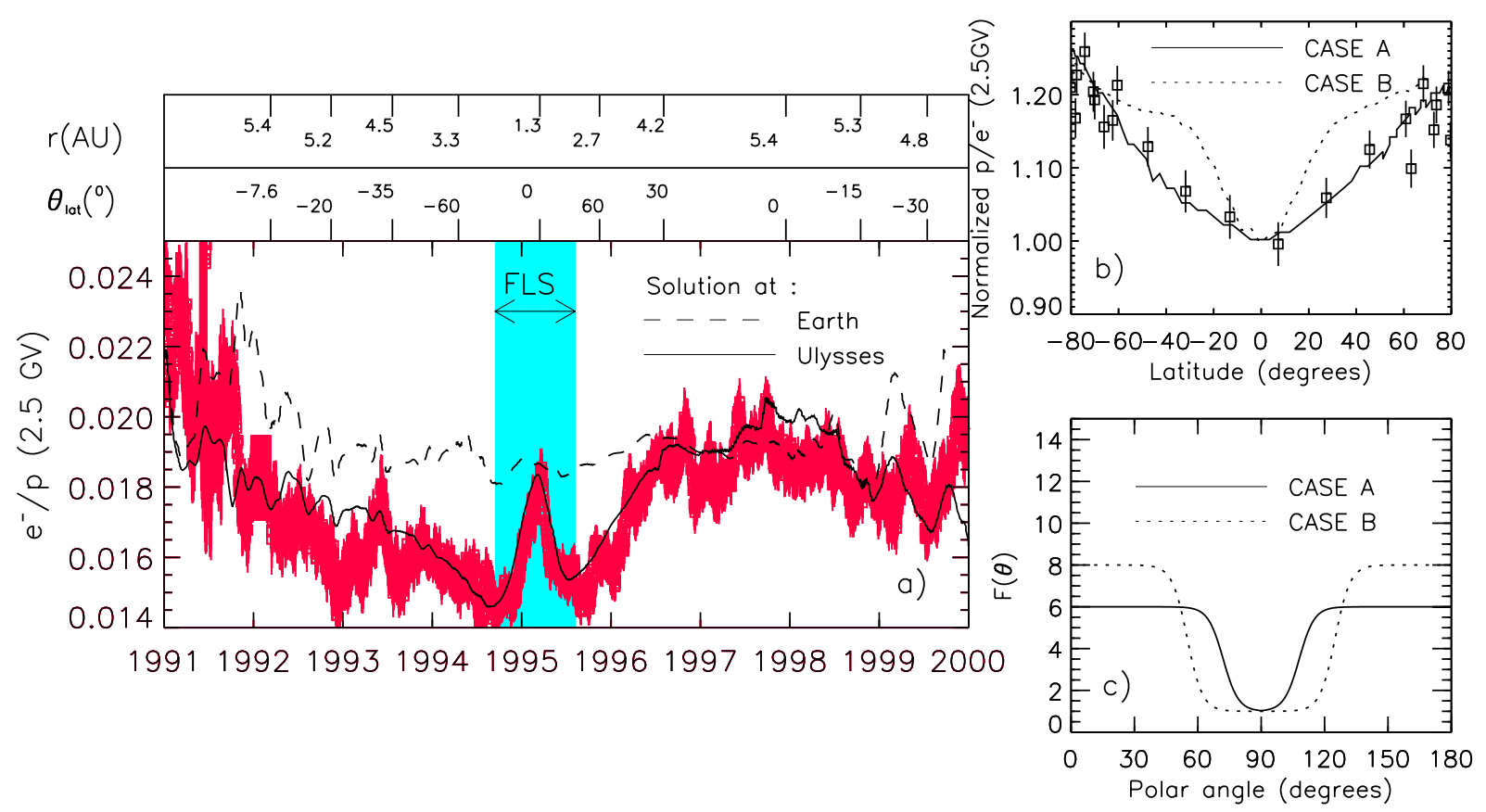

Fig. 4. (a) Computed $2.5 \mathrm{GV}$ electron to proton ratio (e/p) along Ulysses' trajectory (solid line) and at Earth (dotted line) in comparison with the $2.5 \mathrm{GV}$ e/p observations from KET (Heber et al., 1996; 1999; 2002). (b) Two computed proton-to-electron ratios (p/e) as a function of Ulysses' latitude for the fast latitude scan period around 1995 in comparison with the observed p/e from KET. CASE A and CASE B correspond to two assumptions of $K_{\perp \theta}$ in Eq. (3) by changing $F(\theta)$ as a function of polar angle shown in (c); CASE B, dotted line, corresponds to $d=8$ and $\theta_{F}=35^{\circ}$ and CASE A, solid line, to $d=6$ and $\theta_{F}=15^{\circ}$ in Eq. (3).

of charge-sign-dependence caused by controlled drifts are, therefore, well understood for solar minimum and intermediate activity periods. However, for solar maximum periods the modelling is below the observations. This aspect was fully investigated by Ferreira (2002), who concentrated on particle drifts at and during extreme solar maximum conditions - see also Ferreira et al. (2003). The difference between the electron-to-proton ratio along the Ulysses' trajectory and at Earth during the fast latitude is indicative of the drift-related latitude dependence of the protons but not for the electrons during this period.

Looking into greater detail on a shorter time scale, Fig. 4b highlights the latitude dependence of $2.5 \mathrm{GV}$ protons during Ulysses' fast latitude scan period. Shown here are the observed, normalized proton-to-electron ratios and the computed p/e as a function of Ulysses' latitude (Heber et al., 1996; 1999; 2002). Two computed scenarios, normalized in the equatorial plane, are compared to the observed values corresponding to two assumptions of $K_{\perp \theta}$ in Eq. (3) done through different $F(\theta)$, as shown in Fig. 4c. CASE B is computed with $d=8$ and $\theta_{F}=35^{\circ}$, also previously used by Burger et al. (2000) and Ferreira et al. (2001), while for CASE A, $d=6$ and $\theta_{F}=15^{\circ}$. For CASE B the model yields a strong latitudinal dependence close to the equatorial plane but much less over the poles, inconsistent with the observations. To rectify the situation, $F(\theta)$ are changed to CASE A, which illustrates that the enhancement of $K_{\perp \theta}$ should be somewhat less and that it must occur closer to the equato- rial plane for this period than previously assumed. Evidently, CASE A produces excellent agreement with the observed $\mathrm{p} / \mathrm{e}$ at solar minimum as an illustration of the important role perpendicular diffusion plays, in particular the fact that it must be anisotropic in the sense of having a significant enhancement towards the polar regions of the heliosphere.

\section{Summary and conclusions}

Charge-sign dependent modulation is one of the important features of cosmic ray modulation because it is the most direct indication of gradient, curvature and current sheet drifts in the heliosphere. Studying it along Ulysses' trajectory emphasizes the difference in the latitude dependence of protons and electrons at $2.5 \mathrm{GV}$ caused by drifts. In this work we also emphasize how the observed charge-sign modulation effect for $1.2 \mathrm{GV}$ electron and Helium over 22 years can be explained. For this the time-dependent variation of all the diffusion coefficients and drifts was determined by applying the compound approach to long-term modulation. These calculations illustrate quantitatively how diffusion increases and how drifts decrease from solar minimum to maximum modulation, as required to make the model compatible to the observations. In addition to the time-dependence of the diffusion coefficients, a significant larger perpendicular diffusion toward the polar region is required to reduce the large latitudinal effects caused by unmodified drifts. 
The compound approach to modelling long-term modulation of cosmic intensities at Earth has proved to be remarkably successful as a comparison to data over a period of 22 years shown. Apart from describing the modulation of cosmic rays over these long periods, the model also produces realistic charge-sign dependent modulation from minimum to maximum solar activity, and can straightforwardly account for the latitude dependence of cosmic ray protons and the lack thereof for electrons along Ulysses' trajectory. This approach contributes to the improved understanding of how diffusion and drifts may vary from solar minimum to maximum modulation.

Acknowledgements. We thank Adri Burger, Gerd Wibberenz, Bill Webber and the participants of the Potchefstroom Cosmic Ray Workshop in March 2002 for interesting discussions on longterm/charge-sign dependent modulation. The ULYSSES/KET project is supported under grant No. 50 ON 9103 by the German Bundesminister für Bildung und Forschung (BMBF) through the Deutsches Zentrum für Luft und Raumfahrt (DLR). We also thank the South African National Research Foundation for financial support.

Topical Editor R. Forsyth thanks two referees for their help in evaluating this paper.

\section{References}

Burger, R. A., Hattingh, M., and Bieber, J. W.: The effect of magnetic helicity on the propagation of galactic cosmic rays, Adv. Space Res., 19, 897-901, 1997.

Burger, R. A. and Potgieter, M. S.: The effect of large current sheet tilt angles in numerical modulation models: a theoretical assessment, Proc. 26th Int. Cos. Ray Conf, 7, 13-17, 1999.

Burger, R. A., Potgieter, M. S., and Heber, B.: Rigidity dependence of cosmic-ray proton latitudinal gradients measured by the Ulysses spacecraft: Implications for the diffusion tensor, J. Geophys. Res., 105, 27 447-27 456, 2000.

Burlaga, L. F., McDonald, F. B., and Ness, N. F.: Cosmic ray modulation and the distant heliospheric magnetic field: Voyager 1 and 2 observations from 1986 to 1989, J. Geophys. Res., 98, 1-11, 1993.

Cane, H. V., Wibberenz, G., Richardson, I. G., and von Rosenvinge, T. T.: Cosmic ray modulation and the solar magnetic field, Geophys. Res. Lett., 26, 565-568, 1999.

Clem, J. M., Clements, D. P., Esposito, J., Evenson, P., Huber, D., et al.: Solar modulation of cosmic electrons, Astrophys. J., 464, 507-515, 1996.

Clem, J. M., Evenson, P., and Heber, B.: Cosmic electron gradients in the inner heliosphere, Geophys. Res. Lett., 29, 11-1-11-4, 2002 .

Cummings, A. C. and Stone, E. C.: Onset of solar modulation in the outer heliosphere as seen in anomalous cosmic rays, Proc. 27th Inter. Cosmic Ray Conf., 10, 4251-4255, 2001.

Evenson, P.: Cosmic ray electrons, Space Sci. Rev., 83, 63-73, 1998.

Ferreira, S. E. S.: The heliospheric transport of galactic cosmic rays and jovian electrons, Ph.D. thesis, Potchefstroom University, South Africa, 2002.

Ferreira, S. E. S. and Potgieter, M. S.: Galactic cosmic rays in the heliosphere, Adv. Space Res., in press, 2003.
Ferreira, S. E. S. and Potgieter, M. S.: On the tilt angles of the heliospheric current sheet used in modulation models over a 22year cosmic ray modulation cycle, Adv. Space Res., in press, 2003.

Ferreira, S. E. S., Potgieter, M. S., and Moeketsi, D. M.: Modulation effects of a changing solar wind speed on low energy electrons, Adv. Space Res., in press, 2003.

Ferreira, S. E. S., Potgieter, M. S., Burger, R. A., and Heber, B.: Modulation effects of anisotropic perpendicular diffusion on $\mathrm{CR}$ electron intensities in the heliosphere, J. Geophys. Res., 105, 18 305-18 314, 2000.

Ferreira, S. E. S., Potgieter, M. S., Burger, R. A., Heber, B., and Fichtner, H.: Modulation of jovian and galactic electrons in the heliosphere 1. Latitudinal transport of a few-MeV electrons, J. Geophys. Res., 106, 24 979-24 988, 2001.

Fisk, L. A.: Motion of the foot points of heliospheric magnetic field lines at the Sun: Implications for recurrent energetic particle events at high heliographic latitudes, J. Geophys. Res., 101, 15 547-15 554, 1996.

Giacalone, J. and Jokipii, J. R.: The transport of cosmic rays across a turbulent magnetic field, Astrophys. J., 520, 204-214, 1999.

Haasbroek, L. J. and Potgieter, M. S.: The modulation of cosmic rays in the high latitude heliosphere: A computer simulation, Space Sci. Rev., 72, 385-390, 1995.

Heber, B. and Potgieter, M. S.: Galactic cosmic ray observations at different heliospheric latitudes, Adv. Space Res., 26, 839-852, 2000.

Heber B., Dröge, W., Ferrando, P., Haasbroek, L. J., Kunow, H., Müller-Mellin, R., Paizis, C., Potgieter, M. S., Raviart, A., and Wibberenz, G.: Spatial variation of $>40 \mathrm{MeV} / \mathrm{n}$ nuclei fluxes observed during the Ulysses rapid latitude scan, Astron. Astrophys. 316, 538-546, 1996.

Heber, B., Wibberenz, G., Potgieter, M. S., Burger, R. A.: Ulysses Cosmic Ray and Solar Particle Investigation/Kiel Electron Telescope observations: Charge-sign dependence and spatial gradients during the 1990-2000 A > 0 polarity magnetic cycle, J. Geophys. Res., 107, SSH2-1, 2002.

Heber, B., Ferrando, P., Raviart, A., Wibberenz, G., et al.: Differences in temporal variations of galactic cosmic ray electrons and protons: Implications from Ulysses at solar minimum, Geophys. Res. Lett., 26, 2133-2136, 1999.

Hoeksema, J. T.: Large scale structure of the heliospheric magnetic field; 1976-1991, in; Solar Wind Seven, (Eds) Marsch, E. and Schwenn, R., Pergamon, Oxford, 191-196, 1992.

Jokipii, J. R, Levy, E. H., and Hubbard, W. B.: Effects of particle drift on cosmic ray transport. I General properties and application to solar modulation, Astrophys. J., 213, 861-868, 1977.

Jokipii, J. R. and Kóta, J.: The polar heliospheric magnetic field, Geophys. Res. Lett., 16, 1-4, 1989.

Kissmann, R., Fichtner, H., Heber, B., Ferreira, S. E. S., and Potgieter, M. S.: First results of a new 3D model of the timedependent modulation of elctrons in the heliosphere, Adv. Space. Res., 2003.

Kóta, J. and. Jokipii, J. R: Modelling of 3-D corotating cosmic ray structures in the heliosphere, Space Sci. Rev., 83, 137-145, 1998.

le Roux, J. A. and Potgieter, M. S.: A numerical model for a cosmicray modulation barrier in the outer heliosphere, Astron. Astrophys., 209, 406-410, 1989.

le Roux, J. A. and Potgieter, M. S.: The simulation of complete 11 and 22 year modulation cycles for CRs in the heliosphere using a drift model with global merged interaction regions, Astrophys. J., 442, 847-851, 1995. 
le Roux, J. A., Zank, G. P., and Ptuskin, V. S.: An evaluation of perpendicular diffusion models regarding cosmic ray modulation on the basis of a hydromagnetic description for solar wind turbulence, J. Geophys. Res., 104, 24 845-24 862, 1999.

McComas, D. J., Goldstein, R., Gosling, J. T., and Skoug, R. M.: Ulysses' second orbit: remarkably different solar wind, Space Sci. Rev., 97, 99-103, 2001.

McDonald, F. B.: Cosmic-ray modulation in the heliosphere, Space Sci. Rev., 83, 33-50, 1998.

McDonald, F. B., Fuji, Z., Ferrando, P., Heber, B., Raviart, A., Kunow, H., et al.: The cosmic ray radial and latitudinal intensity gradients in the inner and outer heliosphere 1996-2001.3, Proc. 27th Inter. Cosmic Ray Conf., 10, 3906-3910, 2001.

McKibben, R. B., Simpson, J. A., Zhang, M., Bame, S., and Balogh, A.: Ulysses Out-of-Ecliptic Observations of "27-day" Variations in High Energy Cosmic Ray Intensity, Space Sci. Rev., 72, 403408, 1995.

Paizis, C., Heber, B., Ferrando, P., Raviart, A., Falconi, B., Marzolla, S., Potgieter, M. S., Bothmer, V., Kunow, H., MüllerMellin, R., and Posner, A.: Amplitude evolution and rigidity dependence of the 26-day recurrent decreases: COSPIN/KET results, J. Geophys. Res., 104, 28 241-28 247, 1999.

Parker, E. N.: The passage of energetic charged particles through interplanetary space, Planet. Space Sci., 13, 9-49, 1965.

Perko, J. S. and Fisk, L. A.: Solar modulation of galactic cosmic rays. 5. Time dependent modulation, J. Geophys. Res., 88, 90339036, 1983.

Potgieter, M. S.: Time-dependent cosmic-ray modulation: role of drifts and interaction regions, Adv. Space Res., 13, 239-243, 1993.

Potgieter, M. S.: The long-term modulation of galactic cosmic rays in the heliosphere, Adv. Space Res., 16, 191-195, 1995.
Potgieter M. S.: The heliospheric modulation of galactic cosmic rays at solar minimum and beyond, Adv. Space Res. 19, 883887, 1997.

Potgieter, M. S.: The heliospheric modulation of cosmic ray protons: Role of enhanced perpendicular diffusion during periods of minimum solar modulation, J. Geophys. Res., 105, $18295-$ $18304,2000$.

Potgieter, M. S. and Ferreira, S. E. S.: Modulation of cosmic rays in the heliosphere over 11 and 22 year cycles: A modeling perspective, Adv. Space Res., 27(3), 481-492, 2001.

Potgieter, M. S. and le Roux, J. A.: The long-term heliospheric modulation of galactic cosmic rays according to a timedependent drift model with merged interaction regions, Astrophys. J., 423, 817-827, 1994.

Potgieter, M. S., Burger, R. A., and Ferreira, S. E. S.: Modulation of cosmic rays in the heliosphere from solar minimum to maximum: a theoretical perspective, Space Sci. Rev., 97, 295-307, 2001.

Potgieter, M. S., Haasbroek, L. J., Heber, B., and Ferrando, P.: The modelling of the latitude dependence of cosmic ray protons and electrons in the inner heliosphere, Adv. Space Res., 19(6), 901905, 1997.

Potgieter, M. S., le Roux, J. A., Burlaga, L. F., and McDonald, F. B.: The role of merged interaction regions and drifts in the heliospheric modulation of cosmic rays beyond $20 \mathrm{AU}$ : A computer simulation, Astrophys. J., 403, 760-768, 1993.

Reinecke, J. P. L., Moraal, H., and McDonald, F. B.: The cosmic radiation in the heliosphere at successive solar minima 3. Steadystate drift solutions of the transport equation, J. Geophys. Res., 101, 21 581-21 588, 1996.

Wibberenz, G. and Cane, H. V.: Simple analytical solutions for propagating diffusion barriers and application to the $1974 \mathrm{mini}$ cycle, J. Geophys. Res., 105, 18 315-18 326, 2000. 